\title{
Accurate and Simple Source Localization Using Differential Received Signal Strength
}

\author{
Lanxin $\operatorname{Lin}^{\mathrm{a}}$, H. C. So ${ }^{\mathrm{a}}$, Y. T. Chan ${ }^{\mathrm{b}}$ \\ ${ }^{a}$ Department of Electronic Engineering, City University of Hong Kong, Hong Kong SAR, \\ China \\ ${ }^{b}$ Department of Electrical and Computer Engineering, Royal Military College of Canada, \\ Kingston, Ontario, Canada
}

\begin{abstract}
Locating an unknown-position source using received signal strength (RSS) measurements in an accurate and low-complexity manner is addressed in this paper. Given that the source transmit power is unknown, we employ the differential RSS information to devise two computationally attractive localization methods based on the weighted least squares (WLS) approach. The main ingredients in the first algorithm development are to obtain the unbiased estimates of the squared ranges and introduce an extra variable. The second method improves the first version by implicitly exploiting the relationship between the extra variable and source location through a second WLS step. The performance of the two estimators is analyzed in the presence of zero-mean white Gaussian disturbances. Numerical examples are also included to evaluate their localization accuracy by comparing with the maximum likelihood approach and Cramér-Rao lower bound.
\end{abstract}

Keywords:

source localization, received signal strength, positioning algorithm, least squares

\section{Introduction}

Source localization refers to finding the position of a target of interest based on measurements from an array of spatially separated sensors with a priori known locations. This research topic has many important applications in radar, sonar, telecommunications, mobile communications and 
wireless sensor network (WSN). Time-of-arrival (TOA), time-difference-ofarrival (TDOA), angle-of-arrival (AOA) and received signal strength (RSS) are standard measurements for positioning [1]-[2]. The TOA, TDOA and RSS contain the range information between the source and sensor while the AOA provides the source bearing relative to the receiver. Compared with TOA and TDOA schemes which require clock synchronization among the sensors and even the source, and with AOA-based localization where an antenna array is needed at each sensor, employing the RSS measurements is more cost effective. In fact, RSS-based positioning is particularly important in sensor network applications where each sensor has limited computational and communication capabilities and thus it is desirable to locate the source with minimum cost in terms of both hardware and software [3].

Maximum likelihood (ML) [4], semi-definite relaxation (SDR) [5], centroid [6] and weighted least squares (WLS) [7, 8, 9] approaches are the common solutions for source localization using RSS measurements. It has been shown in [4] that the positioning accuracy of the ML methodology attains CramérRao lower bound (CRLB) at sufficiently small noise conditions. However, the ML cost function is highly nonlinear. Hence its maximization is sensitive to initial conditions and there is no guarantee of global optimality. The basic idea of the SDR technique is to approximate the nonconvex ML formulation to a convex optimization program which always guarantees a global solution. Generally speaking, when the relaxation is sufficiently tight, the SDR solution is an approximate ML estimate, but its computational complexity is very high. In centroid localization, the source location is approximated as the weighted average of all receivers within its transmission range. Although it is simple to implement and robust to variations in the propagation environment, its positioning accuracy is generally low. On the other hand, the WLS approach converts the RSS measurements to a set of linear equations in the source position, resulting in a computationally simple solution. Assuming that the source transmit power is known, we have recently devised a WLS RSS-based positioning algorithm [9] whose performance can achieve the CRLB in the presence of white Gaussian disturbances. Nevertheless, to the best of our knowledge, there is no optimum WLS estimator for the more challenging scenario of unknown source transmit power in the literature. In this work, we assume that the propagation model, path-loss exponent and shadowing variances are known a priori through a calibration phase. For example, all of them can be measured prior to the deployment of nodes in a WSN localization application. However, the transmit power will be subject 
to large uncertainty even if the node specification is available. It is because its value is very dependent on the height and orientation of the node. Other factors affecting the transmit power include tolerances in the transmitter components, losses in RF connectors and power supply voltage variations. To eliminate the transmit power uncertainty, we utilize here the differential RSS measurements to devise accurate and fast WLS-based positioning algorithms. Note that in cognitive radio applications, the transmission power is also unknown [10].

The rest of the paper is organized as follows. In Section 2, the problem formulation with the RSS signal model is first introduced. To avoid dealing with the unknown nuisance parameter of the transmit power, we employ the differential RSS measurements in our algorithm development. After obtaining the unbiased estimates of the squared range ratios and introducing an extra variable, a simple WLS RSS-based position estimator is devised and analyzed. The performance of the proposed method is improved by exploiting the constraint between the source position and the extra variable, and its derivation as well as analysis are provided in Section 3. Simulation results are presented in Section 4 to evaluate the localization accuracy of the proposed approach by comparing with the ML method and CRLB. Finally, conclusions are drawn in Section 5.

\section{Basic Algorithm}

The notations used in this paper are first introduced as follows. The symbols in bold upper case represent matrices and the bold lower case symbols denote vectors. The $M \times 1$ vector $\mathbf{1}_{M}$ has all elements $1, \mathbf{I}_{M}$ is the $M \times M$ identity matrix, and $\mathbf{0}_{M \times N}$ is the $M \times N$ zero matrix. The $[\mathbf{a}]_{i}$ represents the $i$ th element of vector $\mathbf{a}$ and $[\mathbf{A}]_{i, j}$ denotes the $(i, j)$ entry of matrix $\mathbf{A}$. The diag and sgn denote the diagonal and signum functions, respectively. The ${ }^{T}$ is the transpose operator, $\operatorname{Tr}(\cdot)$ is the trace operator, and $\operatorname{rank}(\cdot)$ is the rank operator. In addition, $\mathbb{E}(\cdot)$ and $\operatorname{cov}(\cdot)$ represent the expectation and covariance operators, respectively. The $\overline{\mathbf{A}}$ is the noise-free component in $\mathbf{A}$, and $\widehat{\mathbf{a}}$ and $\tilde{\mathbf{a}}$ denote the estimate and variable of $\mathbf{a}$, respectively. Finally, $q \sim \mathcal{N}\left(\mu, \sigma^{2}\right)$ means that the random variable $q$ follows a Gaussian distribution with mean $\mu$ and variance $\sigma^{2}$.

Since multipath fading can be smoothed out by averaging the RSS measurements over frequency and time while the shadow fading cannot be re- 
moved, the RSS path-loss model is [7]:

$$
P_{i}=P_{s}-10 \gamma \log _{10} d_{i}+n_{i}, i=1,2, \cdots, N
$$

where $P_{i}$ is the averaged signal strength in $\mathrm{dB}$ received at the $i$ th sensor, $P_{s}$ is the unknown source transmit power in $\mathrm{dB}, \gamma$ is the known path-loss factor, $d_{i}$ is the distance between the unknown-position source and $i$ th receiver, and $\left\{n_{i}\right\}$ are the average shadow fadings which are modeled as uncorrelated zero-mean Gaussian variables with known variances $\left\{\sigma_{i}^{2}\right\}$. Note that since $P_{i}$ is the averaged RSS, the averaging reduces the measurement noise to an insignificant level compared to the shadow fading effects. The number of receiver is $N \geq 4$. For simplicity but without loss of generality, we consider two-dimensional positioning and

$$
d_{i}=\sqrt{\left(x-x_{i}\right)^{2}+\left(y-y_{i}\right)^{2}}
$$

where $\mathbf{x}=\left[\begin{array}{ll}x & y\end{array}\right]^{T}$ is the unknown source location and $\left(x_{i}, y_{i}\right)$ are the known coordinates of the $i$ th receiver. The task is to find $\mathbf{x}$ given the $N$ RSS measurements of (1). To avoid estimating $P_{s}$, we use the first receiver as the reference and convert (1) to the differential RSS model:

$$
P_{i, 1}^{\prime}=-\gamma \ln \left(\frac{d_{i}}{d_{1}}\right)+m_{i, 1}, i=2,3, \cdots, N
$$

where $P_{i, 1}^{\prime}=0.1 \ln (10)\left(P_{i}-P_{1}\right)$ and $m_{i, 1}=0.1 \ln (10)\left(n_{i}-n_{1}\right)$.

Our algorithm development is based on the linearization approach which requires $d_{i}^{2}$. In order to get an estimate of $d_{i}^{2}$, we first express (3) as:

$$
e^{-\frac{2}{\gamma} P_{i, 1}^{\prime}}=\left(\frac{d_{i}}{d_{1}}\right)^{2} e^{-\frac{2}{\gamma} m_{i, 1}}, i=2,3, \cdots, N
$$

where the noise component is now multiplicative. From [11], the expected value of (4) is

$$
\mathbb{E}\left\{e^{-\frac{2}{\gamma} P_{i, 1}^{\prime}}\right\}=\left(\frac{d_{i}}{d_{1}}\right)^{2} e^{\frac{2}{\gamma^{2}}\left(\lambda_{i}^{2}+\lambda_{1}^{2}\right)}
$$

where $\lambda_{i}^{2}=0.01(\ln (10))^{2} \sigma_{i}^{2}$. Based on (4) and (5), we get an unbiased estimate of $d_{i}^{2} / d_{1}^{2}$, denoted by $r_{i, 1}$ :

$$
r_{i, 1}=e^{-\frac{2}{\gamma} P_{i, 1}^{\prime}-\frac{2}{\gamma^{2}}\left(\lambda_{i}^{2}+\lambda_{1}^{2}\right)}, i=2,3, \cdots, N
$$


For sufficiently small noise conditions, we then have:

$$
r_{i, 1} d_{1}^{2} \approx d_{i}^{2}, \quad i=2,3, \cdots, N
$$

Following [12], we introduce a variable $R=x^{2}+y^{2}$ to express $d_{i}^{2}$ as:

$$
d_{i}^{2}=R-2 x_{i} x-2 y_{i} y+x_{i}^{2}+y_{i}^{2}, \quad i=1,2, \cdots, N
$$

Substituting (8) into (7) yields a set of linear equations:

$$
\mathbf{A} \boldsymbol{\theta}=\mathbf{b}+\mathbf{w}
$$

where

$$
\begin{gathered}
\mathbf{A}=\left[\begin{array}{ccc}
2 x_{2}-2 r_{2,1} x_{1} & 2 y_{2}-2 r_{2,1} y_{1} & r_{2,1}-1 \\
\vdots & \vdots & \vdots \\
2 x_{N}-2 r_{N, 1} x_{1} & 2 y_{N}-2 r_{N, 1} y_{1} & r_{N, 1}-1
\end{array}\right] \\
\mathbf{b}=\left[\begin{array}{c}
x_{2}^{2}+y_{2}^{2}-r_{2,1}\left(x_{1}^{2}+y_{1}^{2}\right) \\
\vdots \\
x_{N}^{2}+y_{N}^{2}-r_{N, 1}\left(x_{1}^{2}+y_{1}^{2}\right)
\end{array}\right]
\end{gathered}
$$

$\boldsymbol{\theta}=\left[\begin{array}{ll}\mathbf{x} R\end{array}\right]^{T}$ and $\mathbf{w}=\left[\begin{array}{lll}w_{2} \cdots w_{N}\end{array}\right]^{T}$ is the error vector. From (7), it is clear that $w_{i}=d_{1}^{2} r_{i, 1}-d_{i}^{2}, i=2,3, \cdots, N$, with $\mathbb{E}\{\mathbf{w}\}=\mathbf{0}_{(N-1) \times 1}$ according to (5).

Without considering the dependency between $\mathbf{x}$ and $R$, the WLS estimate of $\boldsymbol{\theta}$ for (9), which is also the best linear unbiased estimator, is [13]:

$$
\widehat{\boldsymbol{\theta}}=\left(\mathbf{A}^{T} \mathbf{C}_{\mathbf{w}}^{-1} \mathbf{A}\right)^{-1} \mathbf{A}^{T} \mathbf{C}_{\mathbf{w}}^{-1} \mathbf{b}
$$

where $\mathbf{C}_{\mathbf{w}}=\operatorname{cov}(\mathbf{w})$. In Appendix $A$, it is proved that

$$
\mathrm{C}_{\mathrm{w}}=\Sigma \Lambda \Sigma
$$

where

$$
\boldsymbol{\Lambda}=\left[\begin{array}{cccc}
e^{\frac{4}{\gamma^{2}}\left(\lambda_{2}^{2}+\lambda_{1}^{2}\right)}-1 & e^{\frac{4}{\gamma^{2}} \lambda_{1}^{2}}-1 & \cdots & e^{\frac{4}{\gamma^{2}} \lambda_{1}^{2}}-1 \\
\vdots & \vdots & \ddots & \vdots \\
e^{\frac{4}{\gamma^{2}} \lambda_{1}^{2}}-1 & e^{\frac{4}{\gamma^{2}} \lambda_{1}^{2}}-1 & \cdots & e^{\frac{4}{\gamma^{2}}\left(\lambda_{N}^{2}+\lambda_{1}^{2}\right)}-1
\end{array}\right]
$$


and $\boldsymbol{\Sigma}=\operatorname{diag}\left(d_{2}^{2}, \cdots, d_{N}^{2}\right)$. As scaling of $\mathbf{C}_{\mathbf{w}}$ does not affect the solution in (10), we can use $\boldsymbol{\Sigma}=\operatorname{diag}\left(d_{2}^{2} / d_{1}^{2}, \cdots, d_{N}^{2} / d_{1}^{2}\right)$. For sufficiently small noise conditions, we replace $d_{i}^{2} / d_{1}^{2}$ by $r_{i, 1}$ to yield a practical form of $\mathbf{C}_{\mathbf{w}}$ :

$$
\mathrm{C}_{\mathrm{w}} \approx \Sigma^{\prime} \Lambda \Sigma^{\prime}
$$

where $\boldsymbol{\Sigma}^{\prime}=\operatorname{diag}\left(r_{2,1}, \cdots, r_{N, 1}\right)$ according to $(7)$. The position estimate is thus given as $\widehat{\mathbf{x}}=\left[[\widehat{\boldsymbol{\theta}}]_{1}[\widehat{\boldsymbol{\theta}}]_{2}\right]^{T}$.

In Appendix $\mathrm{B}$, we have proved that the covariance for $\widehat{\boldsymbol{\theta}}$ is

$$
\mathbf{C}_{\widehat{\boldsymbol{\theta}}} \approx\left(\overline{\mathbf{A}}^{T} \mathbf{C}_{\mathbf{w}}^{-1} \overline{\mathbf{A}}\right)^{-1}
$$

As a result, the theoretical root mean square error (RMSE) for $\widehat{\mathbf{x}}$, denoted by $\operatorname{RMSE}(\widehat{\mathbf{x}})$, is

$$
\operatorname{RMSE}(\widehat{\mathbf{x}})=\sqrt{\left[\mathbf{C}_{\widehat{\boldsymbol{\theta}}}\right]_{1,1}+\left[\mathbf{C}_{\widehat{\boldsymbol{\theta}}}\right]_{2,2}}
$$

\section{Improved Version}

Following [9] and [12], a second WLS step which utilizes the relationship between the unknown position $\mathbf{x}$ and extra variable $R$, can increase the estimation performance. Based on $x^{2}+y^{2}=R$ and under sufficiently small noise conditions such that $x \approx[\widehat{\boldsymbol{\theta}}]_{1}$ and $y \approx[\widehat{\boldsymbol{\theta}}]_{2}$, we can construct:

$$
\mathrm{Gz}=\mathbf{h}+\mathbf{q}
$$

where

$$
\begin{gathered}
\mathbf{G}=\left[\begin{array}{lll}
1 & 0 & 1 \\
0 & 1 & 1
\end{array}\right]^{T} \\
\mathbf{z}=\left[x^{2} y^{2}\right]^{T} \\
\mathbf{h}=\left[[\widehat{\boldsymbol{\theta}}]_{1}^{2}[\widehat{\boldsymbol{\theta}}]_{2}^{2}[\widehat{\boldsymbol{\theta}}]_{3}\right]^{T}
\end{gathered}
$$


and

$$
\mathbf{q}=\left[\begin{array}{c}
x^{2}-[\widehat{\boldsymbol{\theta}}]_{1}^{2} \\
y^{2}-[\widehat{\boldsymbol{\theta}}]_{2}^{2} \\
R-[\widehat{\boldsymbol{\theta}}]_{3}
\end{array}\right]=\left[\begin{array}{c}
\left(x+[\widehat{\boldsymbol{\theta}}]_{1}\right)\left(x-[\widehat{\boldsymbol{\theta}}]_{1}\right) \\
\left(y+[\widehat{\boldsymbol{\theta}}]_{2}\right)\left(y-[\widehat{\boldsymbol{\theta}}]_{2}\right) \\
R-[\widehat{\boldsymbol{\theta}}]_{3}
\end{array}\right] \approx\left[\begin{array}{c}
2 x\left(x-[\widehat{\boldsymbol{\theta}}]_{1}\right) \\
2 y\left(y-[\widehat{\boldsymbol{\theta}}]_{2}\right) \\
R-[\widehat{\boldsymbol{\theta}}]_{3}
\end{array}\right]
$$

The WLS estimate of $\mathbf{z}$ is

$$
\widehat{\mathbf{z}}=\left(\mathbf{G}^{T} \mathbf{C}_{\mathbf{q}}^{-1} \mathbf{G}\right)^{-1} \mathbf{G}^{T} \mathbf{C}_{\mathbf{q}}^{-1} \mathbf{h}
$$

where $\mathbf{C}_{\mathbf{q}}=\operatorname{cov}(\mathbf{q})=\operatorname{diag}(2 x, 2 y, 1) \mathbf{C}_{\widehat{\boldsymbol{\theta}}} \operatorname{diag}(2 x, 2 y, 1)$. Since $x, y$, and $\overline{\mathbf{A}}$ are not available, we employ $x \approx[\widehat{\boldsymbol{\theta}}]_{1}, y \approx[\widehat{\boldsymbol{\theta}}]_{2}$ and $\mathbf{C}_{\widehat{\boldsymbol{\theta}}} \approx\left(\mathbf{A}^{T} \mathbf{C}_{\mathbf{w}}^{-1} \mathbf{A}\right)^{-1}$ in the actual implementation.

As (16) are the estimates of $x^{2}$ and $y^{2}$, we employ the sign information of (10) to find $(x, y)$. Fusing (10) and (16), the improved position estimate, denoted by $\widehat{\mathbf{x}}^{o}$, is:

$$
\widehat{\mathbf{x}}^{o}=\left[\operatorname{sgn}\left([\widehat{\boldsymbol{\theta}}]_{1}\right) \sqrt{[\widehat{\mathbf{z}}]_{1}} \operatorname{sgn}\left([\widehat{\boldsymbol{\theta}}]_{2}\right) \sqrt{[\widehat{\mathbf{Z}}]_{2}}\right]^{T}
$$

To derive the covariance of $\widehat{\mathbf{x}}^{o}$, we first notice that (17) can be viewed as minimizing the WLS cost function constructed from (9) subject to $[\boldsymbol{\theta}]_{1}^{2}+$ $[\boldsymbol{\theta}]_{2}^{2}=[\boldsymbol{\theta}]_{3}$, and thus the corresponding cost function is:

$$
\begin{aligned}
J(\widetilde{\mathbf{x}}) & =\left(\left[\mathbf{A}_{1} \mathbf{a}\right]\left[\begin{array}{c}
\widetilde{\mathbf{x}} \\
\widetilde{\mathbf{x}}^{T} \widetilde{\mathbf{x}}
\end{array}\right]-\mathbf{b}\right)^{T} \mathbf{C}_{\mathbf{w}}^{-1}\left(\left[\mathbf{A}_{1} \mathbf{a}\right]\left[\begin{array}{c}
\widetilde{\mathbf{x}} \\
\widetilde{\mathbf{x}}^{T} \widetilde{\mathbf{x}}
\end{array}\right]-\mathbf{b}\right) \\
& =\left(\mathbf{A}_{1} \widetilde{\mathbf{x}}+\mathbf{a} \widetilde{\mathbf{x}}^{T} \widetilde{\mathbf{x}}-\mathbf{b}\right)^{T} \mathbf{C}_{\mathbf{w}}^{-1}\left(\mathbf{A}_{1} \widetilde{\mathbf{x}}+\mathbf{a} \widetilde{\mathbf{x}}^{T} \widetilde{\mathbf{x}}-\mathbf{b}\right)
\end{aligned}
$$

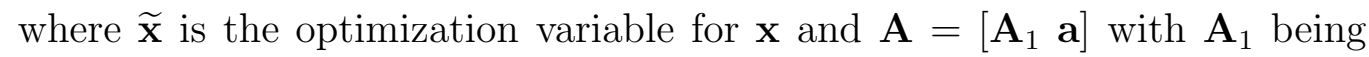
the first two columns of $\mathbf{A}$ and $\mathbf{a}$ is its last column. Applying the covariance formula for unconstrained optimization problems at sufficiently small noise conditions [14]-[15] yields:

$$
\left.\mathbf{C}_{\widehat{\mathbf{x}}^{o}} \approx[\mathbb{E}\{\mathbf{H}(J(\widetilde{\mathbf{x}}))\}]^{-1} \mathbb{E}\left\{\nabla(J(\widetilde{\mathbf{x}})) \nabla^{T} J(\widetilde{\mathbf{x}})\right\}[\mathbb{E}\{\mathbf{H}(J(\widetilde{\mathbf{x}}))\}]^{-1}\right|_{\widetilde{\mathbf{x}}=\mathbf{x}}
$$

where $\mathbf{H}(J(\widetilde{\mathbf{x}}))=\partial^{2} J(\widetilde{\mathbf{x}}) / \partial \widetilde{\mathbf{x}} \partial \widetilde{\mathbf{x}}^{T}$ and $\nabla(J(\widetilde{\mathbf{x}}))=\partial J(\widetilde{\mathbf{x}}) / \partial \widetilde{\mathbf{x}}$ are the corresponding Hessian matrix and gradient vector, respectively. In Appendix C, we have derived $\mathbf{C}_{\widehat{\mathbf{x}}^{o}}$ as:

$$
\mathbf{C}_{\widehat{\mathbf{x}}^{o}} \approx\left(\mathbf{B}^{T} \mathbf{C}_{\mathbf{m}}^{-1} \mathbf{B}\right)^{-1}
$$


where

$$
\begin{aligned}
\mathbf{B} & =\frac{5 \gamma}{\ln (10)} \boldsymbol{\Sigma}^{-1}\left(\mathbf{A}_{1}+2 \mathbf{a x}^{T}\right) \\
& =-\frac{10 \gamma}{\ln (10)}\left[\begin{array}{cc}
\frac{x-x_{2}}{d_{2}^{2}}-\frac{x-x_{1}}{d_{1}^{2}} & \frac{y-y_{2}}{d_{2}^{2}}-\frac{y-y_{1}}{d_{1}^{2}} \\
\vdots & \vdots \\
\frac{x-x_{N}}{d_{N}^{2}}-\frac{x-x_{1}}{d_{1}^{2}} & \frac{y-y_{N}}{d_{N}^{2}}-\frac{y-y_{1}}{d_{1}^{2}}
\end{array}\right]
\end{aligned}
$$

and $\mathbf{C}_{\mathbf{m}}=\mathbb{E}\left\{\mathbf{m m}^{T}\right\}=\operatorname{diag}\left(\sigma_{2}^{2}, \cdots, \sigma_{N}^{2}\right)+\sigma_{1}^{2} \mathbf{1}_{N-1} \mathbf{1}_{N-1}^{T}$ with $\mathbf{m}=\left[m_{2,1} m_{3,1}\right.$ $\left.\cdots m_{N, 2}\right]^{T}$. It is also proved in Appendix $\mathrm{C}$ that (20) is equal to the CRLB based on the RSS measurements of (1), indicating the optimality of (17). That is to say, the optimum solution derived from (1), which estimates both the position and transmit power, provides the same localization accuracy as that of (3). Nevertheless, it seems that finding $(x, y)$ from $(3)$ is simpler than employing (1).

\section{Numerical Examples}

Computer simulations are carried out to evaluate the RMSE performance of the proposed WLS and two-step WLS algorithms by comparing with the ML method. The ML approach [4] is implemented using the LevenbergMarquardt (LM) scheme with a damping factor of 10 and 20 iterations, which is initialized by $\widehat{\mathbf{x}}$ of (10). In all simulation settings, the LM method converges to a minimum within 20 iterations. Note that apart from employing the number of iterations, we can also exploit the difference between successive estimates as the stopping criterion. Unless stated otherwise, the path-loss factor is $\gamma=4$ and there are eight receivers, that is, $N=8$, and their coordinates are $(0,0),(10,0),(10,10),(0,10),(5,0),(10,5),(5,10)$ and $(0,5)$, which are all located on the boundary of a $10 \times 10$ area. The shadow fadings $\left\{n_{i}\right\}$ are zero-mean white Gaussian variables with known identical variances of $\sigma_{i}^{2}=\sigma^{2}$. All results are averages of 5000 independent runs.

Figure 1 shows the RMSEs of the WLS and ML methods at $\mathbf{x}=\left[\begin{array}{ll}3 & 7\end{array}\right]^{T}$ for $\sigma \in[-10,10] \mathrm{dB}$. The theoretical standard deviation for $\widehat{\mathbf{x}}$, namely, (14), and that of the two-step WLS estimator, which is also the square root of the CRLB, are included. The performance of the standard WLS estimator agrees with (14) for the whole range of $\sigma$. On the other hand, the RMSEs of both two-step WLS estimator and ML scheme attain $\sqrt{\text { CRLB }}$ although the latter 
is sightly superior for $\sigma \leq 5 \mathrm{~dB}$. Regarding implementation complexity, the average computation times per trial for the WLS, improved WLS and ML methods are $1.7 \times 10^{-4} \mathrm{~s}, 2.7 \times 10^{-4} \mathrm{~s}$ and $2.1 \times 10^{-3} \mathrm{~s}$, respectively, indicating the computational attractiveness of the proposed approach. The RMSEs for different $\gamma \in[1,5]$ at $\sigma=5 \mathrm{~dB}$ are plotted in Figure 2. The WLS algorithms attain their theoretical standard deviations when $\gamma>2$ while the ML method is optimal in the whole range of $\gamma$. It is seen that although the RSS decreases with $\gamma$, the localization accuracy increases with $\gamma$, which is also indicated by the CRLB. Figure 3 shows the RMSEs at $\sigma=5 \mathrm{~dB}$ when the number of receivers varies from $N=4$ to $N=8$. That is, for each $N$, the $N$ receivers which are closest to $\mathbf{x}$ are employed. It is seen that the performance of all methods improves as $N$ increases. In particular, the two-step scheme is comparable with the ML estimator and the performance of all methods deviates from their theoretical calculations at $N=4$.

The first test is repeated when the unknown-position source is randomly located within the $10 \times 10$ area in each trial, and the results are plotted in Figure 4. We see that the RMSE of $\widehat{\mathbf{x}}$ is around 1.5 times of that of $\widehat{\mathbf{x}}^{o}$, which is similar to Figure 1. The RMSEs of the proposed WLS and two-step WLS methods also meet their theoretical standard deviations for sufficiently small noise conditions but the ML approach cannot provide reliable performance. Note that some RMSEs of the ML estimator are very large and thus are not included in the plot. It is because the ML cost function is multimodal and the LM algorithm cannot guarantee a global minimization. Even when initialized by the WLS estimate of (10), which is close to the global solution, the LM scheme still converges occasionally to a local minimum. The only way to achieve the global optimality is to perform a dense grid search but its computational complexity is very demanding. We also repeat the first test for $\mathbf{x}=\left[\begin{array}{ll}-3 & 7\end{array}\right]^{T}$, which is located outside the square bounded by the sensor coordinates, and the results are plotted in Figure 5. It is observed that the standard and two-step WLS algorithms can attain their theoretical performance when $\sigma \leq-2 \mathrm{~dB}$ and $\sigma \leq-5 \mathrm{~dB}$, respectively, indicating that they are also effective in locating a source outside the convex hull. On the other hand, the ML method is only optimal when $\sigma$ is close to $-10 \mathrm{~dB}$.

In both the WLS and ML algorithms, $\sigma^{2}$ is assumed known. However, in many situations, $\sigma^{2}$ is only approximately known, and its value can also vary with time. The next experiment examines the sensitivity of the algorithms to deviations in $\sigma^{2}$ from the assumed value. That is, now we only have an estimate of $\sigma^{2}$ for each RSS measurement, which is modeled as $\hat{\sigma}^{2} \sim$ 
$\mathcal{N}\left(\sigma^{2}, 0.3 \sigma^{2}\right)$. Repeating the simulations of Figure 1 but with uncertainty in the value of $\sigma^{2}$ gives the RMSE in Figure 6. Under this condition, the two-step WLS solution is more robust than ML estimator which exhibits large errors at some instances. Note that some RMSEs of the ML estimator are very large and thus are not included in the plot. When $\sigma^{2}$ is sufficiently small, Appendix $\mathrm{C}$ shows that $r_{i, 1} \approx e^{-\frac{2}{\gamma} P_{i, 1}^{\prime}}$, that is, uncertainty in $\sigma^{2}$ has only a small impact on $\mathbf{A}$ and $\mathbf{b}$, and the estimation accuracy.

Finally, the performance of the investigated positioning algorithms is evaluated using the real RSS data collected in the measurement campaign reported in [1]. In the data set, there are RSS measurements between 44 known-position sensors in an office environment as well as the estimated path-loss factor and noise powers. Although the transit power $P_{s}$ is also provided, we do not utilize this information. When estimating each sensor position, we use 43 RSS measurements from the remaining receivers, that is, non-cooperative localization is assumed. The root squared error (RSE) performance of the WLS and ML algorithms is plotted in Figure 7. Moreover, the average RSEs over the 44 sensors of the standard WLS, two-step WLS and ML methods are measured as $2.3 \mathrm{~m}, 1.8 \mathrm{~m}$ and $1.4 \mathrm{~m}$, respectively. These indicate that the WLS approaches are comparable with the ML scheme in terms of localization accuracy. Note that the RSE comes from a single trial, which is different from the statistical RMSE. Hence it is quite conceivable that the two-step WLS and ML methods can produce a higher RSE than the suboptimal WLS estimator.

\section{Conclusion}

Two received signal strength (RSS)-based positioning algorithms are developed using the weighted least squares (WLS) approach, which changes nonlinear equations to linear equations via the introduction of an intermediate variable. For unknown source transmit power, the first algorithm is a standard WLS technique which utilizes unbiased estimates of the squared ranges. The second estimator improves the first version by implicitly exploiting the constraint between the source location and the intermediate variable. The mean square errors of the two WLS methods are derived and confirmed by computer simulations. In particular, the localization accuracy of the im-

proved algorithm attains Cramér-Rao lower bound under sufficiently small noise conditions. 


\section{Appendix A}

The covariance matrix of (11) is derived as follows. According to (9), the elements of $\mathbf{C}_{\mathbf{w}}$ are:

$$
\begin{aligned}
{\left[\mathbf{C}_{\mathbf{w}}\right]_{i, j}=} & \mathbb{E}\left\{\left(d_{1}^{2} r_{i+1,1}-d_{i+1}^{2}\right)\left(d_{1}^{2} r_{j+1,1}-d_{j+1}^{2}\right)\right\} \\
=d_{1}^{4} \mathbb{E}\left\{r_{i+1,1} r_{j+1,1}\right\}-d_{1}^{2} d_{i+1}^{2} \mathbb{E}\left\{r_{j+1,1}\right\}-d_{1}^{2} d_{j+1}^{2} \mathbb{E}\left\{r_{i+1,1}\right\}+d_{i+1}^{2} d_{j+1}^{2}, & \\
& \quad i, j=1,2, \cdots, N-1
\end{aligned}
$$

From (3), we have

$$
\begin{aligned}
-2 / \gamma P_{i, 1}^{\prime} & \sim \mathcal{N}\left(2 \ln \left(d_{i} / d_{1}\right), 4 / \gamma^{2}\left(\lambda_{i}^{2}+\lambda_{1}^{2}\right)\right) \\
-2 / \gamma\left(P_{i, 1}^{\prime}+P_{j, 1}^{\prime}\right) & \sim \mathcal{N}\left(2\left(\ln \left(d_{i} / d_{1}\right)+\ln \left(d_{j} / d_{1}\right)\right), 4 / \gamma^{2}\left(\lambda_{i}^{2}+\lambda_{j}^{2}+4 \lambda_{1}^{2}\right)\right), i \neq j
\end{aligned}
$$

It is well known [16] that if $q$ is a Gaussian variable with mean $\mu$ and variance $\sigma^{2}$, then $E\left\{e^{q}\right\}=e^{\mu+\sigma^{2} / 2}$ and $\operatorname{var}\left(e^{q}\right)=\left(e^{\sigma^{2}}-1\right) e^{2 \mu+\sigma^{2}}$. As a result, the expected values of $r_{i, 1}^{2}$ and $r_{i, 1} r_{j, 1}$ are

$$
\mathbb{E}\left\{r_{i, 1}^{2}\right\}=\frac{d_{i}^{4}}{d_{1}^{4}} e^{\frac{4}{\gamma^{2}}\left(\lambda_{i}^{2}+\lambda_{1}^{2}\right)}
$$

and

$$
\mathbb{E}\left\{r_{i, 1} r_{j, 1}\right\}=\frac{d_{i}^{2} d_{j}^{2}}{d_{1}^{4}} e^{\frac{4}{\gamma^{2}} \lambda_{1}^{2}} \quad i, j=2,3, \cdots, N, i \neq j
$$

Substituting (A.2)-(A.3) into (A.1) with $\mathbb{E}\left\{r_{i, 1}\right\}=d_{i}^{2} / d_{1}^{2}, i=2,3, \cdots, N-1$, and according to (5), we obtain (11).

\section{Appendix B}

The covariance matrix of (13) is derived as follows. We first express $\mathbf{A}=\overline{\mathbf{A}}+\boldsymbol{\Delta}$ where $\boldsymbol{\Delta}=\mathbf{w}\left[-2 x_{1} / d_{1}^{2}-2 y_{1} / d_{1}^{2} 1 / d_{1}^{2}\right]$ is its noise component with $\mathbb{E}\{\boldsymbol{\Delta}\}=\mathbf{0}_{(N-1) \times 3}$. The covariance matrix for $\widehat{\boldsymbol{\theta}}$ is:

$$
\begin{aligned}
\mathbf{C}_{\widehat{\boldsymbol{\theta}}} & =\mathbb{E}\left\{(\widehat{\boldsymbol{\theta}}-\boldsymbol{\theta})(\widehat{\boldsymbol{\theta}}-\boldsymbol{\theta})^{T}\right\} \\
& =\mathbb{E}\left\{\left(\mathbf{A}^{T} \mathbf{C}_{\mathbf{w}}^{-1} \mathbf{A}\right)^{-1} \mathbf{A}^{T} \mathbf{C}_{\mathbf{w}}^{-1} \mathbf{w} \mathbf{w}^{T} \mathbf{C}_{\mathbf{w}}^{-1} \mathbf{A}\left(\mathbf{A}^{T} \mathbf{C}_{\mathbf{w}}^{-1} \mathbf{A}\right)^{-1}\right\}
\end{aligned}
$$


Applying Slutsky's theorem [17], (B.1) is approximated as:

$$
\begin{aligned}
\mathbf{C}_{\widehat{\boldsymbol{\theta}}} & \approx\left[\mathbb{E}\left\{\mathbf{A}^{T} \mathbf{C}_{\mathbf{w}}^{-1} \mathbf{A}\right\}\right]^{-1} \mathbb{E}\left\{\mathbf{A}^{T} \mathbf{C}_{\mathbf{w}}^{-1}\right\} \mathbb{E}\left\{\mathbf{w w}^{T}\right\} \mathbb{E}\left\{\mathbf{C}_{\mathbf{w}}^{-1} \mathbf{A}\right\}\left[\mathbb{E}\left\{\mathbf{A}^{T} \mathbf{C}_{\mathbf{w}}^{-1} \mathbf{A}\right\}\right]^{-1} \\
& =\left[\mathbb{E}\left\{\mathbf{A}^{T} \mathbf{C}_{\mathbf{w}}^{-1} \mathbf{A}\right\}\right]^{-1} \overline{\mathbf{A}}^{T} \mathbf{C}_{\mathbf{w}}^{-1} \overline{\mathbf{A}}\left[\mathbb{E}\left\{\mathbf{A}^{T} \mathbf{C}_{\mathbf{w}}^{-1} \mathbf{A}\right\}\right]^{-1}
\end{aligned}
$$

For sufficiently small noise conditions, $w_{i}=d_{i}^{2}\left(e^{-\frac{2}{\gamma} m_{i, 1}-\frac{2}{\gamma^{2}}\left(\lambda_{i}^{2}+\lambda_{1}^{2}\right)}\right) \approx 0$ and thus $\boldsymbol{\Delta}$ is negligible compared with $\overline{\mathbf{A}}$ element by element, we then have

$$
\mathbb{E}\left\{\mathbf{A}^{T} \mathbf{C}_{\mathbf{w}}^{-1} \mathbf{A}\right\}=\overline{\mathbf{A}}^{T} \mathbf{C}_{\mathbf{w}}^{-1} \overline{\mathbf{A}}+\mathbb{E}\left\{\boldsymbol{\Delta}^{T} \mathbf{C}_{\mathbf{w}}^{-1} \boldsymbol{\Delta}\right\} \approx \overline{\mathbf{A}}^{T} \mathbf{C}_{\mathbf{w}}^{-1} \overline{\mathbf{A}}
$$

Substituting (B.3) into (B.2) yields (13).

\section{Appendix C}

In this Appendix, we first derive (20) and then the CRLB based on the signal model of (1). It is also shown that the CRLBs for (1) and (3) are equivalent. The expected value of Hessian matrix for $J(\widetilde{\mathbf{x}})$ is expressed as:

$$
\left.\mathbb{E}\left\{\frac{\partial^{2} J(\widetilde{\mathbf{x}})}{\partial \widetilde{\mathbf{x}} \widetilde{\mathbf{x}}^{T}}\right\}\right|_{\widetilde{\mathbf{x}}=\mathbf{x}}=\left[\left.\left.\mathbb{E}\left\{\frac{\partial}{\partial \tilde{x}}\left(\frac{\partial J(\widetilde{\mathbf{x}})}{\partial \widetilde{\mathbf{x}}}\right)\right\}\right|_{\widetilde{\mathbf{x}}=\mathbf{x}} \mathbb{E}\left\{\frac{\partial}{\partial \tilde{y}}\left(\frac{\partial J(\widetilde{\mathbf{x}})}{\partial \widetilde{\mathbf{x}}}\right)\right\}\right|_{\widetilde{\mathbf{x}}=\mathbf{x}}\right]
$$

We start with differentiating (18) with respect to $\widetilde{\mathbf{x}}$ to obtain

$$
\frac{\partial J(\widetilde{\mathbf{x}})}{\partial \widetilde{\mathbf{x}}}=2\left(\mathbf{A}_{1}+2 \mathbf{a} \widetilde{\mathbf{x}}^{T}\right)^{T} \mathbf{C}_{\mathbf{w}}^{-1}\left(\mathbf{A}_{1} \widetilde{\mathbf{x}}+\mathbf{a} \widetilde{\mathbf{x}}^{T} \widetilde{\mathbf{x}}-\mathbf{b}\right)
$$

Differentiating (C.2) with respect to $\widetilde{x}$, we get

$$
\begin{aligned}
\frac{\partial}{\partial \tilde{x}}\left(\frac{\partial J(\widetilde{\mathbf{x}})}{\partial \widetilde{\mathbf{x}}}\right)= & 4\left[\begin{array}{ll}
1 & 0
\end{array}\right]^{T} \mathbf{a}^{T} \mathbf{C}_{\mathbf{w}}^{-1}\left(\mathbf{A}_{1} \widetilde{\mathbf{x}}+\mathbf{a} \widetilde{\mathbf{x}}^{T} \widetilde{\mathbf{x}}-\mathbf{b}\right) \\
& +2\left(\mathbf{A}_{1}+2 \mathbf{a} \widetilde{\mathbf{x}}^{T}\right)^{T} \mathbf{C}_{\mathbf{w}}^{-1}\left(\mathbf{A}_{1}\left[\begin{array}{ll}
1 & 0
\end{array}\right]^{T}+2 \mathbf{a} x\right)
\end{aligned}
$$

Putting $\widetilde{\mathbf{x}}=\mathbf{x}$ into (C.3) yields

$$
\begin{aligned}
\left.\frac{\partial}{\partial \tilde{x}}\left(\frac{\partial J(\widetilde{\mathbf{x}})}{\partial \widetilde{\mathbf{x}}}\right)\right|_{\widetilde{\mathbf{x}}=\mathbf{x}}= & 4\left[\begin{array}{ll}
1 & 0
\end{array}\right]^{T} \mathbf{a}^{T} \mathbf{C}_{\mathbf{w}}^{-1}\left(\mathbf{A}_{1} \mathbf{x}+\mathbf{a x}^{T} \mathbf{x}-\mathbf{b}\right) \\
& +2\left(\mathbf{A}_{1}+2 \mathbf{a} \mathbf{x}^{T}\right)^{T} \mathbf{C}_{\mathbf{w}}^{-1}\left(\mathbf{A}_{1}\left[\begin{array}{ll}
1 & 0
\end{array}\right]^{T}+2 \mathbf{a} x\right)
\end{aligned}
$$


Taking expectation on both sides of (C.4) with the use of $\mathbb{E}\{\mathbf{w}\}=\mathbf{0}_{(N-1) \times 1}$, we have:

$$
\left.\mathbb{E}\left\{\frac{\partial}{\partial \tilde{x}}\left(\frac{\partial J(\widetilde{\mathbf{x}})}{\partial \widetilde{\mathbf{x}}}\right)\right\}\right|_{\widetilde{\mathbf{x}}=\mathbf{x}}=2\left(\mathbf{A}_{1}+2 \mathbf{a x}^{T}\right)^{T} \mathbf{C}_{\mathbf{w}}^{-1}\left(\mathbf{A}_{1}\left[\begin{array}{ll}
1 & 0
\end{array}\right]^{T}+2 \mathbf{a} x\right)
$$

Similarly, repeating the derivation of (C.4)-(C.5) with the variable $\tilde{y}$ yields

$$
\left.\mathbb{E}\left\{\frac{\partial}{\partial \tilde{y}}\left(\frac{\partial J(\widetilde{\mathbf{x}})}{\partial \widetilde{\mathbf{x}}}\right)\right\}\right|_{\widetilde{\mathbf{x}}=\mathbf{x}}=2\left(\mathbf{A}_{1}+2 \mathbf{a x}^{T}\right)^{T} \mathbf{C}_{\mathbf{w}}^{-1}\left(\mathbf{A}_{1}\left[\begin{array}{ll}
0 & 1
\end{array}\right]^{T}+2 \mathbf{a} y\right)
$$

Using (C.5)-(C.6), (C.1) becomes:

$$
\left.\mathbb{E}\left\{\frac{\partial^{2} J(\widetilde{\mathbf{x}})}{\partial \widetilde{\mathbf{x}} \widetilde{\mathbf{x}}^{T}}\right\}\right|_{\widetilde{\mathbf{x}}=\mathbf{x}}=2\left(\mathbf{A}_{1}+2 \mathbf{a} \mathbf{x}^{T}\right)^{T} \mathbf{C}_{\mathbf{w}}^{-1}\left(\mathbf{A}_{1}+2 \mathbf{a} \mathbf{x}^{T}\right)
$$

In a similar manner, we obtain:

$$
\left.\mathbb{E}\left\{\frac{\partial J(\widetilde{\mathbf{x}})}{\partial \widetilde{\mathbf{x}}}\left(\frac{\partial J(\widetilde{\mathbf{x}})}{\partial \widetilde{\mathbf{x}}}\right)^{T}\right\}\right|_{\widetilde{\mathbf{x}}=\mathbf{x}}=4\left(\mathbf{A}_{1}+2 \mathbf{a} \mathbf{x}^{T}\right)^{T} \mathbf{C}_{\mathbf{w}}^{-1}\left(\mathbf{A}_{1}+2 \mathbf{a} \mathbf{x}^{T}\right)
$$

Substituting (C.7)-(C.8) into (19) yields:

$$
\mathbf{C}_{\widehat{\mathbf{x}}^{o}} \approx\left[\left(\mathbf{A}_{1}+2 \mathbf{a} \mathbf{x}^{T}\right)^{T} \mathbf{C}_{\mathbf{w}}^{-1}\left(\mathbf{A}_{1}+2 \mathbf{a x} \mathbf{x}^{T}\right)\right]^{-1}
$$

For sufficiently small noise conditions such that

$$
e^{\frac{4}{\gamma^{2}}\left(\lambda_{i}^{2}+\lambda_{1}^{2}\right)} \approx 1+\frac{4\left(\lambda_{i}^{2}+\lambda_{1}^{2}\right)}{\gamma^{2}}=1+\left(\frac{\ln (10)}{5 \gamma}\right)^{2}\left(\sigma_{i}^{2}+\sigma_{1}^{2}\right)
$$

$\mathbf{C}_{\mathbf{w}}^{-1}$ is approximated as:

$$
\mathbf{C}_{\mathbf{w}}^{-1} \approx\left(\frac{5 \gamma}{\ln (10)}\right)^{2} \Sigma^{-1} \mathbf{C}_{\mathbf{m}}^{-1} \boldsymbol{\Sigma}^{-1}
$$

Putting (C.11) into (C.9), we obtain (20). It is noteworthy that (20) is identical to the CRLB for the differential RSS model of (3) [18].

To derive the CRLB for (1), we first compute its corresponding Fisher information matrix (FIM). When the measurement errors are zero-mean Gaussian distributed, the FIM based on RSS measurements of (1), denoted by $\operatorname{FIM}(\boldsymbol{\eta})$, where $\boldsymbol{\eta}=\left[\begin{array}{lll}x & y & P_{s}\end{array}\right]^{T}$, is easily computed as:

$$
\operatorname{FIM}(\boldsymbol{\eta})=\mathbf{D}^{T} \mathbf{C}_{\mathbf{n}}^{-1} \mathbf{D}
$$


where

$$
\mathbf{D}=\frac{\partial \mathbf{f}(\boldsymbol{\eta})}{\partial \boldsymbol{\eta}}=-\frac{10 \gamma}{\ln (10)}\left[\begin{array}{ccc}
\frac{x-x_{1}}{d_{1}^{2}} & \frac{y-y_{1}}{d_{1}^{2}} & -\frac{\ln (10)}{10 \gamma} \\
\vdots & \vdots & \vdots \\
\frac{x-x_{N}}{d_{N}^{2}} & \frac{y-y_{N}}{d_{N}^{2}} & -\frac{\ln (10)}{10 \gamma}
\end{array}\right]
$$

and $\mathbf{C}_{\mathbf{n}}=\operatorname{diag}\left(\sigma_{1}^{2}, \sigma_{2}^{2}, \cdots, \sigma_{N}^{2}\right)$. Since the CRLBs for $x$ and $y$ are given by $\left[\mathrm{FIM}^{-1}(\boldsymbol{\eta})\right]_{1,1}$ and $\left[\mathrm{FIM}^{-1}(\boldsymbol{\eta})\right]_{2,2}$, respectively, we express $\mathrm{FIM}^{-1}(\boldsymbol{\eta})$ as [19]:

$$
\mathrm{FIM}^{-1}(\boldsymbol{\eta})=\left[\begin{array}{cc}
\mathbf{L}^{T} \mathbf{D}^{T} \mathbf{C}_{\mathbf{n}}^{-1} \mathbf{D L} & \mathbf{L}^{T} \mathbf{D}^{T} \mathbf{C}_{\mathbf{n}}^{-1} \mathbf{1}_{N} \\
\mathbf{1}_{N}^{T} \mathbf{C}_{\mathbf{n}}^{-1} \mathbf{D L} & \mathbf{1}_{N}^{T} \mathbf{C}_{\mathbf{n}}^{-1} \mathbf{1}_{N}
\end{array}\right]^{-1}
$$

where $\mathbf{L}^{T}=\left[\begin{array}{ll}\mathbf{I}_{2} & \mathbf{0}_{2 \times 1}\end{array}\right]$. With the use of the partitioned inversion formula and $\mathbf{1}_{N}^{T} \mathbf{C}_{\mathbf{n}}^{-1} \mathbf{1}_{N}=\operatorname{Tr}\left(\mathbf{C}_{\mathbf{n}}^{-1}\right)$, the upper left $2 \times 2$ sub-matrix of $\mathrm{FIM}^{-1}(\boldsymbol{\eta})$, $\mathbf{L}^{T}\left(\mathbf{D}^{T} \mathbf{C}_{\mathbf{n}}^{-1} \mathbf{D}\right)^{-1} \mathbf{L}$, can be computed as

$$
\mathbf{L}^{T}\left(\mathbf{D}^{T} \mathbf{C}_{\mathbf{n}}^{-1} \mathbf{D}\right)^{-1} \mathbf{L}=\left[\mathbf{L}^{T} \mathbf{D}^{T}\left(\mathbf{C}_{\mathbf{n}}^{-1}-\frac{\mathbf{C}_{\mathbf{n}}^{-1} \mathbf{1}_{N} \mathbf{1}_{N}^{T} \mathbf{C}_{\mathbf{n}}^{-1}}{\operatorname{Tr}\left(\mathbf{C}_{\mathbf{n}}^{-1}\right)}\right) \mathbf{D L}\right]^{-1}
$$

To relate (20) and (C.14), we let $\mathbf{P}=\left[-\mathbf{1}_{N-1} \mathbf{I}_{N-1}\right]$ to express $\mathbf{B}$ and $\mathbf{C}_{\mathbf{m}}$ as $\mathbf{B}=\mathbf{P D L}$ and $\mathbf{C}_{\mathbf{m}}=\mathbf{P} \mathbf{C}_{\mathbf{n}} \mathbf{P}^{T}$ so that (20) can be written as:

$$
\mathbf{C}_{\widehat{\mathbf{x}}^{o}}=\left[\mathbf{L}^{T} \mathbf{D}^{T} \mathbf{P}^{T}\left(\mathbf{P} \mathbf{C}_{\mathbf{n}} \mathbf{P}^{T}\right)^{-1} \mathbf{P D L}\right]^{-1}
$$

Using the property of $\mathbf{P} \mathbf{1}_{N}=\mathbf{0}_{(N-1) \times 1}$, we construct an idempotent matrix $\mathbf{S} \in \mathbb{R}^{N \times N}$, which has the form of

$$
\mathbf{S}=\frac{\mathbf{C}_{\mathbf{n}}^{-1 / 2} \mathbf{1}_{N} \mathbf{1}_{N}^{T} \mathbf{C}_{\mathbf{n}}^{-1 / 2}}{\operatorname{Tr}\left(\mathbf{C}_{\mathbf{n}}^{-1}\right)}+\mathbf{C}_{\mathbf{n}}^{1 / 2} \mathbf{P}^{T}\left(\mathbf{P} \mathbf{C}_{\mathbf{n}} \mathbf{P}^{T}\right)^{-1} \mathbf{P C}_{\mathbf{n}}^{1 / 2}
$$

Since $\operatorname{rank}(\mathbf{S})=\operatorname{Tr}(\mathbf{S})=N$, employing the full rank property of $\mathbf{S}$ as well as the idempotent property of $\mathbf{S}\left(\mathbf{I}_{N}-\mathbf{S}\right)=\mathbf{0}_{N \times N}$ yield

$$
\mathbf{S}=\mathbf{I}_{N}
$$

Pre-multiplying and post-multiplying both sides of (C.16) by $\mathbf{C}_{\mathbf{n}}^{-1 / 2}$ and using (C.17), we obtain

$$
\mathbf{P}^{T}\left(\mathbf{P} \mathbf{C}_{\mathbf{n}} \mathbf{P}^{T}\right)^{-1} \mathbf{P}=\mathbf{C}_{\mathbf{n}}^{-1}-\frac{\mathbf{C}_{\mathbf{n}}^{-1} \mathbf{1}_{N} \mathbf{1}_{N}^{T} \mathbf{C}_{\mathbf{n}}^{-1}}{\operatorname{Tr}\left(\mathbf{C}_{\mathbf{n}}^{-1}\right)}
$$


From (C.14), (C.15) and (C.18), we see that the estimation performance of the improved WLS method is equal to CRLB for RSS-based positioning with unknown source transmit power. Furthermore, as the CRLBs for (1) and (3) are identical, there is no performance loss in converting the RSS to differential RSS when $P_{s}$ is unknown.

\section{References}

[1] N. Patwari, A. O. Hero, III, M. Perkins, N. S. Correal, R. J. O’Dea, Relative location estimation in wireless sensor networks, IEEE Transactions on Signal Processing 51 (2003) 2137-2148.

[2] N. Patwari, J. N. Ash, S. Kyperountas, A. O. Hero, III, R. L. Moses, N. S. Correal, Locating the nodes: cooperative localization in wireless sensor networks, IEEE Signal Processing Magazine 22 (2005) 54-69.

[3] F. Hu, X. J. Cao, Wireless Sensor Networks, Boca Raton, FL : Auerbach Publications, 2010.

[4] A. Coluccia, F. Ricciato, On ML estimation for automatic RSS-based indoor localization, in: Proc. IEEE International Symposium on Wireless Pervasive Computing, Modena, Italy, pp. 495-502.

[5] R. W. Ouyang, A. K.-S. Wong, C.-T. Lea, Received signal strengthbased wireless localization via semidefinite programming: noncooperative and cooperative schemes, IEEE Transactions on Vehicular Technology 59 (2010) 1307-1318.

[6] D.-B. Lin, R.-T. Juang, H.-P. Lin, Robust mobile location estimation based on signal attenuation for cellular communication systems, in: Proceedings of the 2005 IEEE Vehicular Technology Conference, volume 4, Stockholm, Sweden, pp. 2425-2428.

[7] J. H. Lee, R. M. Buehrer, Location estimation using differential RSS with spatially correlated shadowing, in: Proc. IEEE Global Telecommunications Conference, Honolulu, HI.

[8] B. C. Liu, K. H. Lin, SSSD-based mobile positioning: On the accuracy improvement issues in distance and location estimations, IEEE Transactions on Vehicular Technology 58 (2009) 1245-1254. 
[9] H. C. So, L. Lin, Linear least squares approach for accurate received signal strength based source localization, IEEE Transactions on Signal Processing 59 (2011) 4035-4040.

[10] S. Kim, H. Jeon, J. Ma, Robust localization with unknown transmission power for cognitive radio, in: Proc. IEEE Military Communications Conference, Florida, USA, pp. 1-6.

[11] S. D. Chitte, S. Dasgupta, Z. Ding, Distance estimation from received signal strength under log-normal shadowing: bias and variance, IEEE Signal Processing Letters 16 (2009) 216-218.

[12] Y. T. Chan, K. C. Ho, A simple and efficient estimator for hyperbolic location, IEEE Transactions on Signal Processing 42 (1994) 1903-1915.

[13] S. M. Kay, Fundamentals of Statistical Signal Processing: Estimation Theory, Englewood Cliffs, NJ: Prentice-Hall, 1993.

[14] K. W. Cheung, H. C. So, A multidimensional scaling framework for mobile location using time-of-arrival measurements, IEEE Transactions on Signal Processing 53 (2005) 460-470.

[15] H. C. So, Source localization: Algorithms and analysis, in: Handbook of Position Location: Theory, Practice and Advances, S.A.Zekavat and M.Buehrer, Eds., Wiley-IEEE Press, pp. 25-66.

[16] E. L. Crow, K. Shimizu (Eds.), Lognormal Distributions : Theory and Applications, New York : Dekker, 1988.

[17] K. C. Ho, Y. T. Chan, Geometric-polar tracking from bearings-only and doppler-bearing measurements, IEEE Transactions on Signal Processing 56 (2008) 5540-5554.

[18] A. K. M. M. Hossain, W. S. Soh, Cramér-Rao bound analysis of localization using signal strength difference as location fingerprint, in: Proc. IEEE INFOCOM, San Diego, CA, pp. 1-9.

[19] F. K. W. Chan, H. C. So, J. Zheng, K. W. K. Lui, Best linear unbiased estimator approach for time-of-arrival based localization, IET Signal Processing 2 (2008) 156-162. 


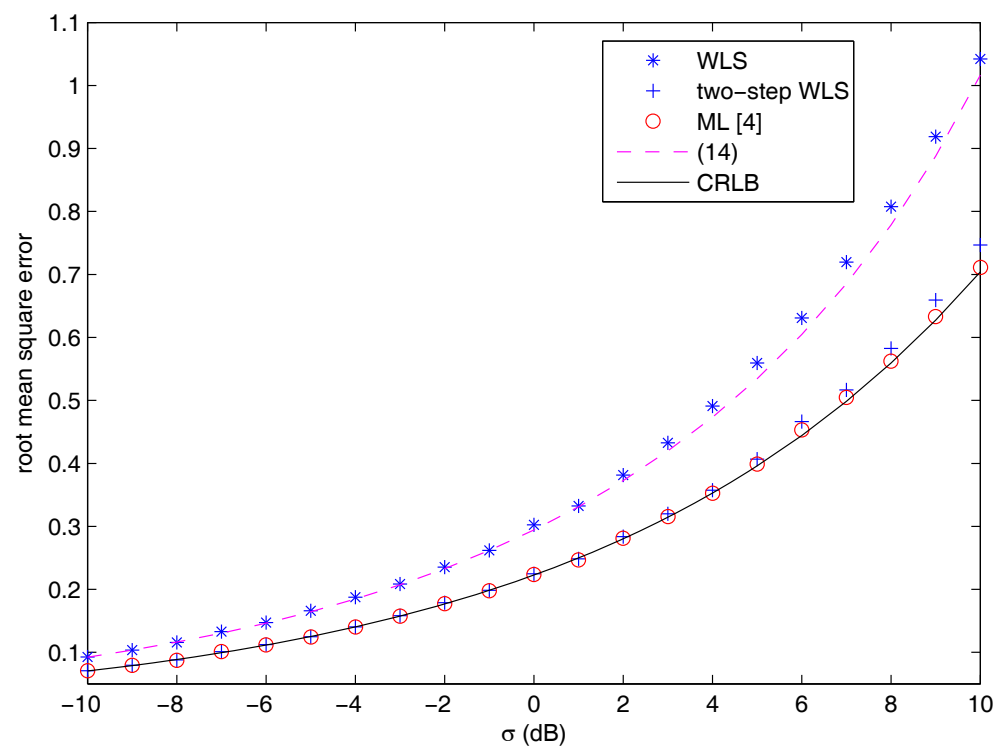

Figure 1: Root mean square error versus $\sigma$ at $\mathbf{x}=[37]^{T}$ when $\gamma=4$ and $N=8$

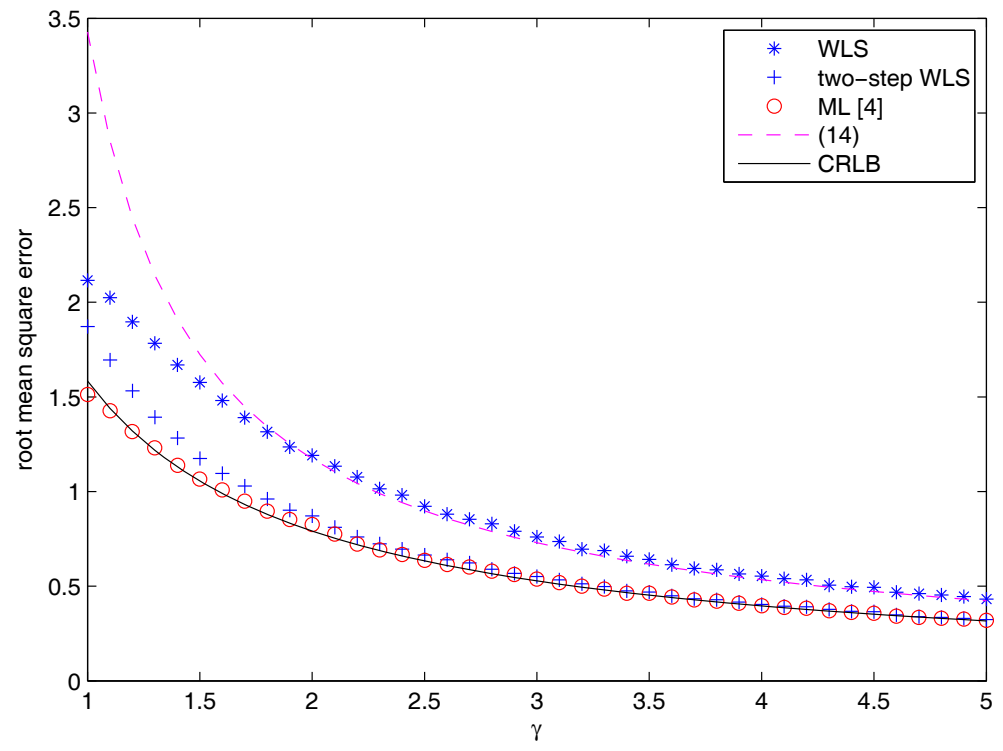

Figure 2: Root mean square error versus $\gamma$ at $\mathbf{x}=\left[\begin{array}{ll}3 & 7\end{array}\right]^{T}$ when $\sigma=5 \mathrm{~dB}$ and $N=8$ 


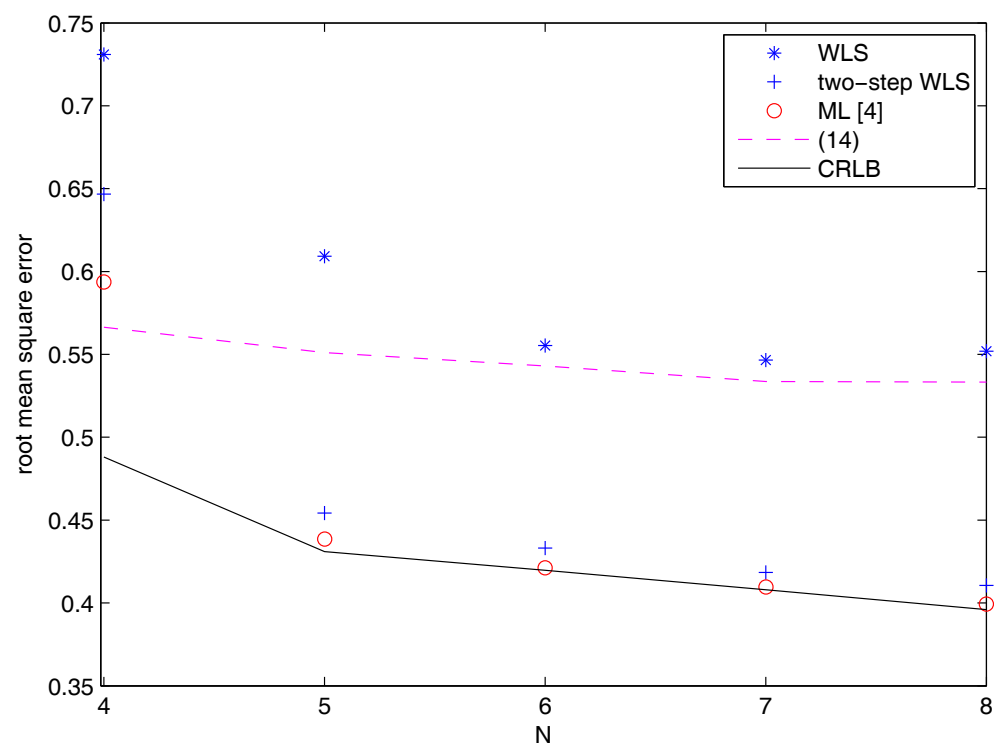

Figure 3: Root mean square error versus $N$ at $\mathbf{x}=\left[\begin{array}{ll}3 & 7\end{array}\right]^{T}$ when $\sigma=5 \mathrm{~dB}$ and $\gamma=4$

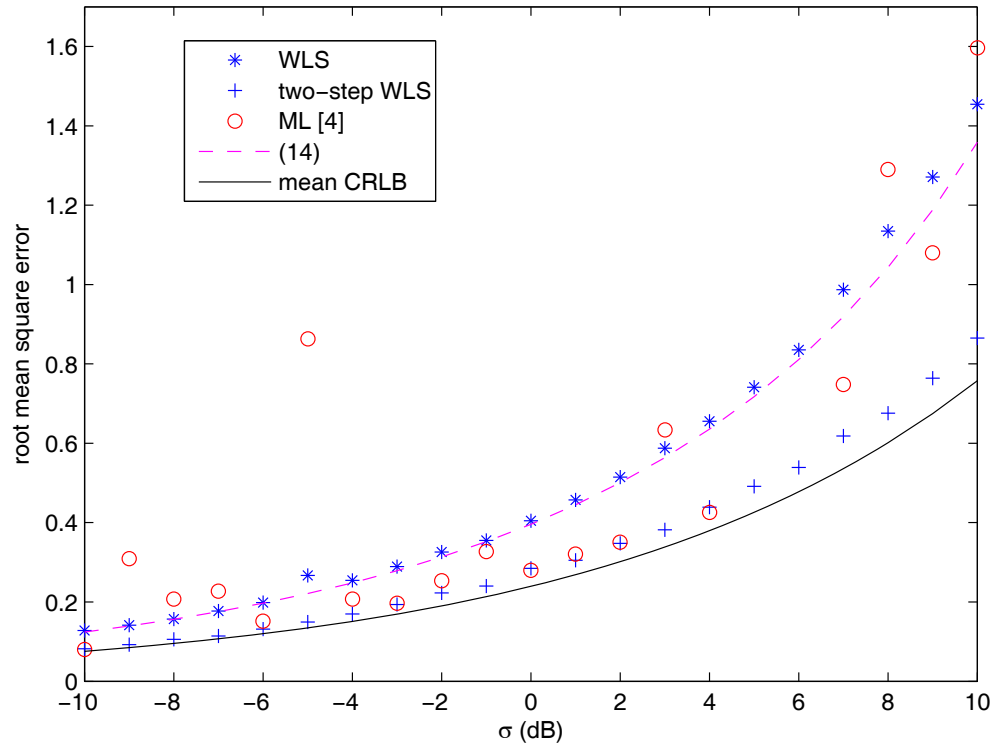

Figure 4: Root mean square error versus $\sigma$ with random source 


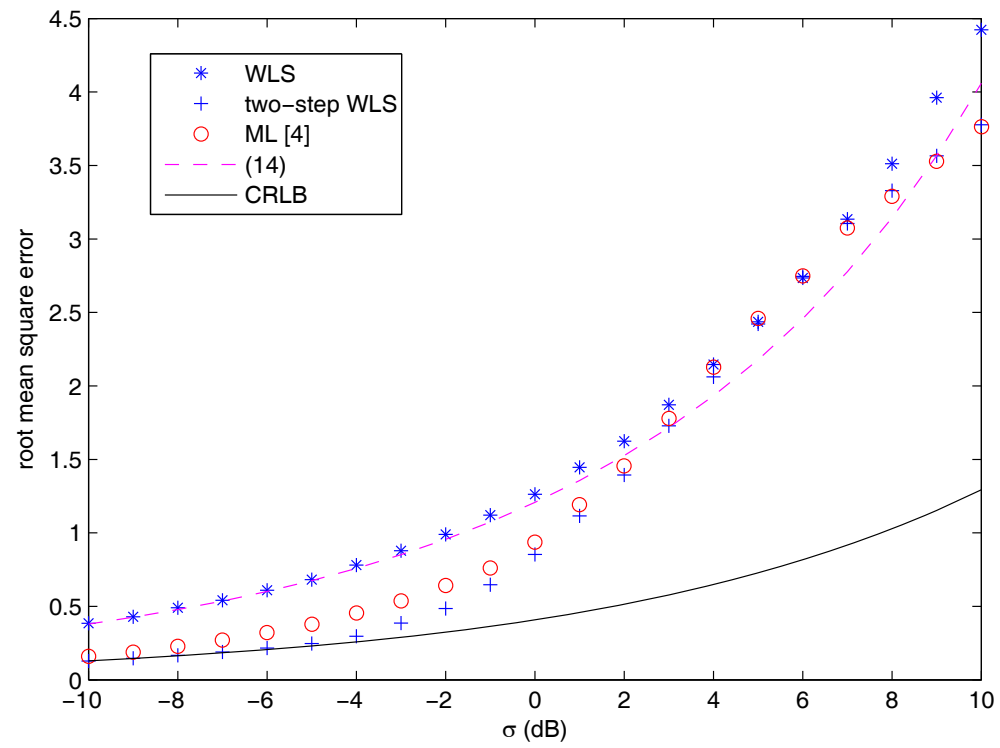

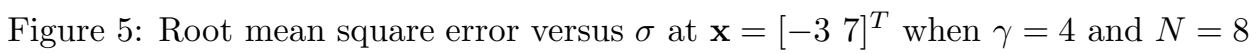

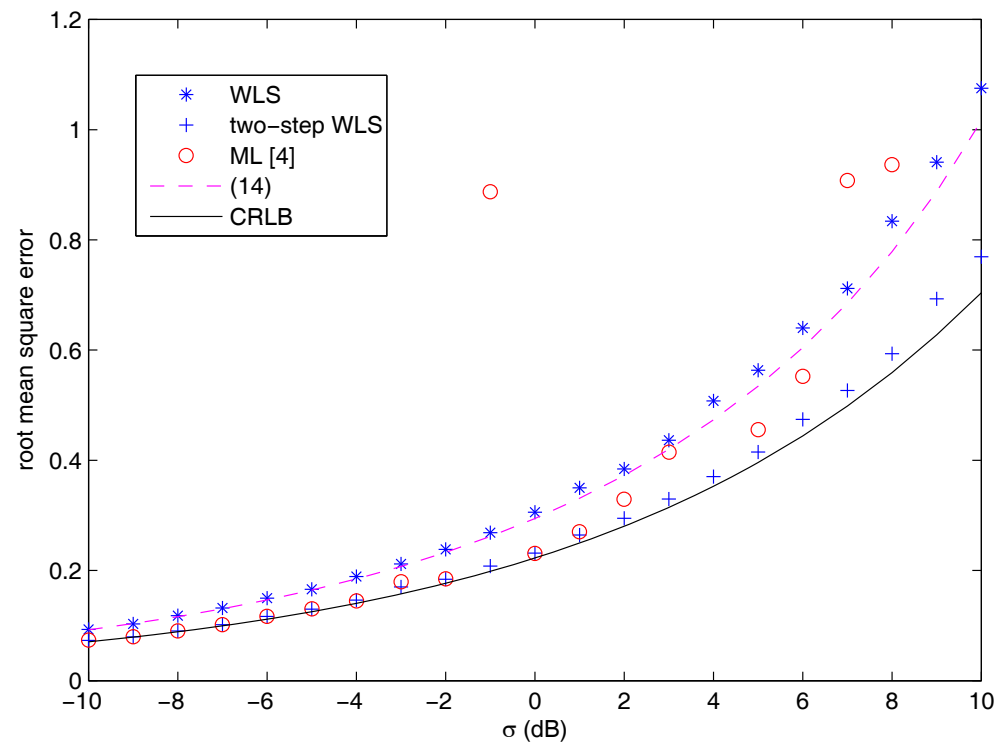

Figure 6: Root mean square error versus $\sigma$ at $\mathbf{x}=\left[\begin{array}{ll}3 & 7\end{array}\right]^{T}$ with noise power uncertainty 


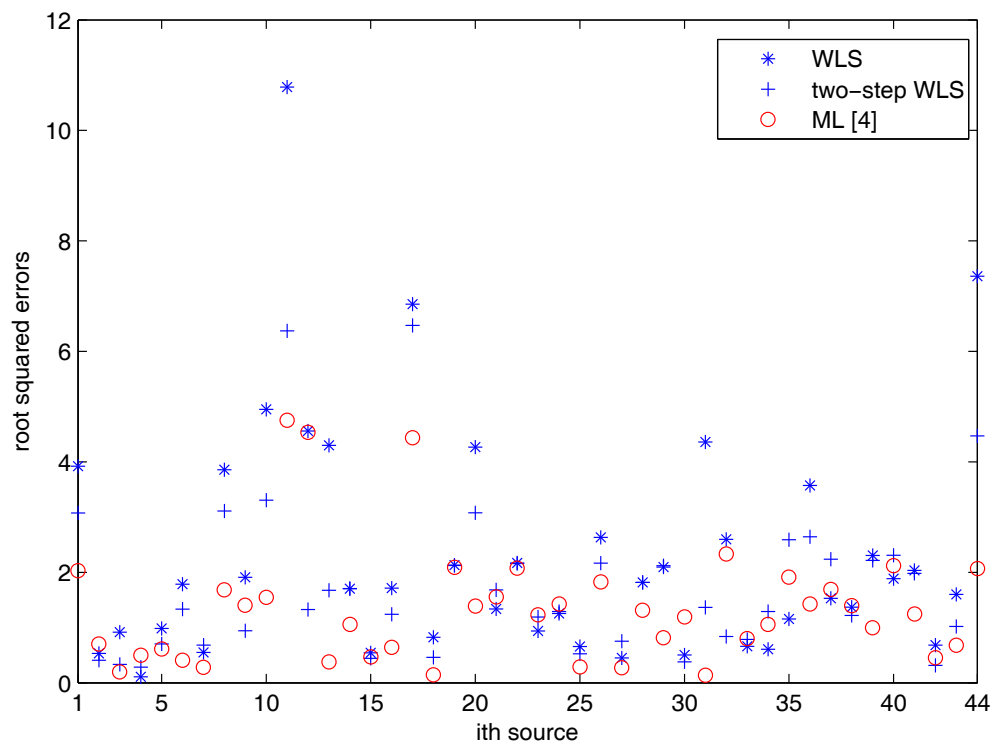

Figure 7: Root squared errors based on real measurements of [1] 